\title{
Leukocytes and Albumin in Canine Leishmaniasis
}

\author{
Társsila Mara Vieira Ferreira', Alexandre Tavares Camelo Oliveira', Victor Machado de Carvalho', \\ Ana Débora Nunes Pinheiro², Thaíse Cristine Ferreira de Carvalho Sombra', Tiago Cunha Ferreira', \\ José Cláudio Carneiro de Freitas ${ }^{1} \&$ Diana Célia Sousa Nunes-Pinheiro ${ }^{1}$
}

\begin{abstract}
Background: Canine Leishmaniasis (CanL) is a multisystemic and chronic inflammatory disease characterized by nonspecific clinical manifestations. In CanL, inflammatory cells and chemical mediators released in response to the parasite play a role in disease development and progression. Alterations on hematological parameters have been documented in CanL. These changes can also be assessed in relation to systemic inflammation caused by this disease. The circulating leukocyte counting, such as neutrophils, as well as the albumin level, are considered direct indicators of an inflammatory host environment. Several studies point to the use of biomarkers on the assistance in diagnosis and prognosis of several canine pathologies. The present study investigated the Neutrophils to Lymphocyte Ratio (NLR), Albumin to Globulin Ratio (AGR), and Neutrophils to Albumin Ratio (NAR) on systemic inflammatory response induced by Canine Leishmaniasis (CanL). Materials, Methods \& Results: For this purpose, adult dogs with confirmed diagnosis to CanL were divided into symptomatic $(\mathrm{SD}, \mathrm{n}=33)$ and asymptomatic (AD, $\mathrm{n}=20)$ dogs for L. infantum and control dogs $(\mathrm{CD}, \mathrm{n}=20)$. Routine hematological and biochemical parameters were determined in blood samples using a veterinary automatic hematology and biochemical analyzers. Asymptomatic dogs (AD) had a higher number of white blood cells and neutrophils $(16.48 \pm 4.93 ; 13.41$ \pm 3.60 , respectively) in relation to symptomatic dogs (SD) $(13.54 \pm 5.13 ; 10.42 \pm 3.69$, respectively) $(P=0.015$ and $P<$ 0.0001 , respectively). Neutrophils to Lymphocyte Ratio (NLR) was higher in dogs with leishmaniasis $(9.45 \pm 3.76)$ than in healthy dogs $(3.39 \pm 1.19)(P<0.0001)$. Serum total proteins $(\mathrm{STP})$ and globulins increased in CanL, while albumin and AGR decreased in CanL, when compared to $\mathrm{CD}$ and references values to canine species. Neutrophils to Albumin Ratio (NAR) was higher in $\mathrm{AD}$ and $\mathrm{SD}(5.02 \pm 1.14 ; 4.79 \pm 1.07$, respectively) when compared to $\mathrm{CD}(2.36 \pm 0.55)(P<0.0001)$. Discussion: As reported in scientific researches, dogs with Leishmaniasis present alterations in circulating cell counts. Based on these data, we decided to expand this information using the NLR as a parameter in an attempt to better clarify the changes in these cells in CanL. We observed that NLR was increased on CanL in relation to healthy dogs, which could be a consequence of relative neutrophilia rather than lymphopenia. Neutrophils to Lymphocyte Ratio (NLR) is a biomarker that conveys information about inflammatory conditions. An elevated NLR can reflect an upregulated innate immune response, since neutrophils are effector cells of innate immunity and are involved in several acute and chronic inflammatory processes. Albumin is an acute phase protein that is considered an immune-inflammatory biomarker, which can be found reduced systemically in progressive inflammatory response. Serum total proteins (STP) and globulins were increased in CanL. These data are already well documented in CanL, which serum globulins are mainly associated with the increase of acute phase proteins, cytokines, and increase of specific antibodies to Leishmania infantum. Our results showed neutrophilia with hypoalbuminemia in CanL. So, in an attempt to assess the relationship of these two available markers, we used NAR calculation in order to evaluate the changes induced by CanL. In this study NAR was higher in CanL when compared to control dogs. Thus, our data indicate that NLR and NAR could be used as biomarkers in veterinary medical clinics in order to assess inflammatory profile in CanL, mainly in asymptomatic dogs. These parameters obtained from routine blood tests might be useful as cost-effective, easily accessible and helpful markers in order to distinguish the inflammatory response intensity in CanL.
\end{abstract}

Keywords: CanL, inflammation, biomarkers, neutrophil, albumin, lymphocyte

DOI: $10.22456 / 1679-9216.111869$

Received: 3 March 2021 Accepted: 21 June 2021 Published: 20 July 2021

${ }^{1}$ Programa de Pós-Graduação em Ciências Veterinárias, Faculdade de Veterinária, Universidade Estadual do Ceará (UECE), Fortaleza, CE, Brazil. ${ }^{2}$ Programa de Pós-Graduação em Química, Universidade Federal Fluminense (UFF), Niterói, RJ, Brazil. CORRESPONDENCE: T.M.V. Ferreira [tarssilamara@outlook.com]. Programa de Pós-Graduação em Ciências Veterinárias - UECE. Av. Dr. Silas Munguba n. 1700. Campus do Itaperi, Bloco C. CEP 60914703 Fortaleza, CE, Brazil. 


\section{INTRODUCTION}

Canine Leishmaniasis (CanL) is a multisystemic disease with a zoonotic character caused by the protozoan Leishmania infantum from the blood meal of infected sandflies [1]. which is characterized by presenting various nonspecific clinical manifestations $[21,45,46]$.

In CanL, the immune response, which is mediated by the inflammatory cells and chemical molecules induced by parasites, plays a role in development and progression of this disease [5,27,38]. The initial immune response involves the migration of phagocytic cells to the infection site $[7,32,42]$, with neutrophils being the most abundant cells with leishmanicidal potential $[7,11,14,30,33]$.

Although alterations in lymphocyte subpopulations and in other hematologic variables have been documented in dogs with CanL $[9,36]$, these changes can also be assessed in relation to systemic inflammation caused by this disease. The use of biomarkers, such as the albumin / globulin ratio (AGR) and the neutrophil / lymphocyte ratio (NLR), proved to be useful in the prognosis of sarcoma [24], mast cell tumor [25], multicentric lymphoma [28], septic peritonitis [17] and other inflammatory conditions in dogs [34]. Another important marker to be evaluated is the Neutrophil to Albumin Ratio (NAR), since the count of circulating leukocytes, such as neutrophils, as well as the albumin level, are considered direct indicators of inflammatory host environment $[13,16,20,23,44]$.

In CanL the use of NLR and NAR as systemic inflammatory biomarkers is unknown. Therefore, the aim of this study was to investigate the role of NLR, AGR, and NAR on the systemic inflammatory response induced by CanL.

\section{MATERIALS AND METHODS}

\section{Animals}

Adult dogs (aged between 3 and 8 years, both sexes) captured by the Zoonoses Control Center with positive serology tests for Leishmania infantum infection (LVC Bio-Manguinhos ${ }^{\circledR}$ DPP associated with ELISA) ${ }^{1}$ were used in this study. Bone marrow aspiration was performed to investigate the amastigote forms of Leishmania spp. Seventy-three dogs were divided in 3 groups [45]: animals without clinical manifestations and with negative results for serological and bone marrow parasitological test for $L$. infantum were considered as control dogs $(C D, n=20)$; asymptomatic dogs $(A D=20)$, which were animals that did not show clinical and laboratorial changes and were positive for infection by L. infantum, and symptomatic $\operatorname{dogs}(\mathrm{SD}=33)$, which were animals with clinical and laboratorial changes for routine testing, and has confirmed infection by L. infantum. It is noteworthy that the infection in these dogs was established by serology and parasitology.

\section{Hematology and biochemical evaluation}

Blood samples (4 mL) were collected by jugular venipuncture and distributed into two tubes, without and with anticoagulants (EDTA-K2). The leukocytes count was performed in a veterinary automatic hematology analyzer ${ }^{2}$. Differential counting of neutrophils, lymphocytes, eosinophils and monocytes was performed on blood smears by a veterinary clinical pathologist under an optical microscope ${ }^{3}$. The Neutrophil to Lymphocyte Ratio (NLR) was calculated according to absolute neutrophil count and the absolute lymphocyte count obtained by routine blood tests. The serum levels of total proteins (STP) and albumin were determined according to the manufacturer's instructions ${ }^{4}$. The globulins were calculated by the difference between the total proteins and albumin values. The Albumin to Globulin Ratio (AGR) was calculated by dividing the concentrations of albumin and globulin, while the Neutrophil to Albumin Ratio (NAR) was calculated according to the absolute neutrophil count and the albumin concentration obtained by routine blood tests. The canine species reference intervals values were used to compare the results.

\section{Statistical analysis}

Data analysis was performed by statistical software $^{5}$. Data with continuous variables were expressed as mean \pm standard deviation and submitted to the Shapiro-Wilk normality test. For non-parametric data as leukocytes, lymphocytes, neutrophils, albumin, globulin, NLR, AGR, NAR, and STP parameters, the Kruskal-Wallis test were used, and for the comparison between groups, the Mann-Whitney test was used. $P$ $<0.05$ was considered significant.

\section{RESULTS}

The inflammatory markers NLR, AGR, NAR, and other parameters were evaluated in symptomatic 
$(\mathrm{SD}=33)$ and asymptomatic $(\mathrm{AD}=20)$ seropositive dogs for $L$. infantum and control dogs $(C D=20)$ [Table 1 and Figure 1].

Initially, the total leukocyte count and cell differentiation were performed. AD had a higher number of WBC and neutrophils (16.48 \pm 4.93 ; $13.41 \pm 3.60$, respectively) in relation to SD (13.54 $\pm 5.13 ; 10.42 \pm 3.69$, respectively) [Table 1]. The results indicated a tendency to neutrophilia with lymphopenia in dogs infected by L. infantum when compared to control dogs. NLR was higher in dogs with leishmaniasis $(9.45 \pm 3.76)$ than healthy dogs $(3.39 \pm 1.19)[P<0.0001]$, and it was noted an increase in NLR of AD $(11.10 \pm 4.36)$ when compared to SD $(8.46 \pm 3.00)(P<0.0001)$ [Table 1 and Figure 1].

Serum total proteins (STP) and globulins levels $(8.38 \pm 1.12 ; 6.11 \pm 0.88$; respectively) were increased in CanL, while albumin and AGR $(2.24 \pm 0.64 ; 0.36$ \pm 0.11 , respectively) were decreased in CanL, when compared to $\mathrm{CD}(6.75 \pm 0.54 ; 3.34 \pm 0.76 ; 3.20 \pm 0.60$; $1.07 \pm 0.58$, respectively) and references values to canine specie (Table 1). NAR presented higher values in $\mathrm{AD}$ and $\mathrm{SD}(5.02 \pm 1.14 ; 4.79 \pm 1.07$, respectively $)$ in comparison to $\mathrm{CD}(2.36 \pm 0.55)(P<0.0001)$ [Table 1 and Figure 1].
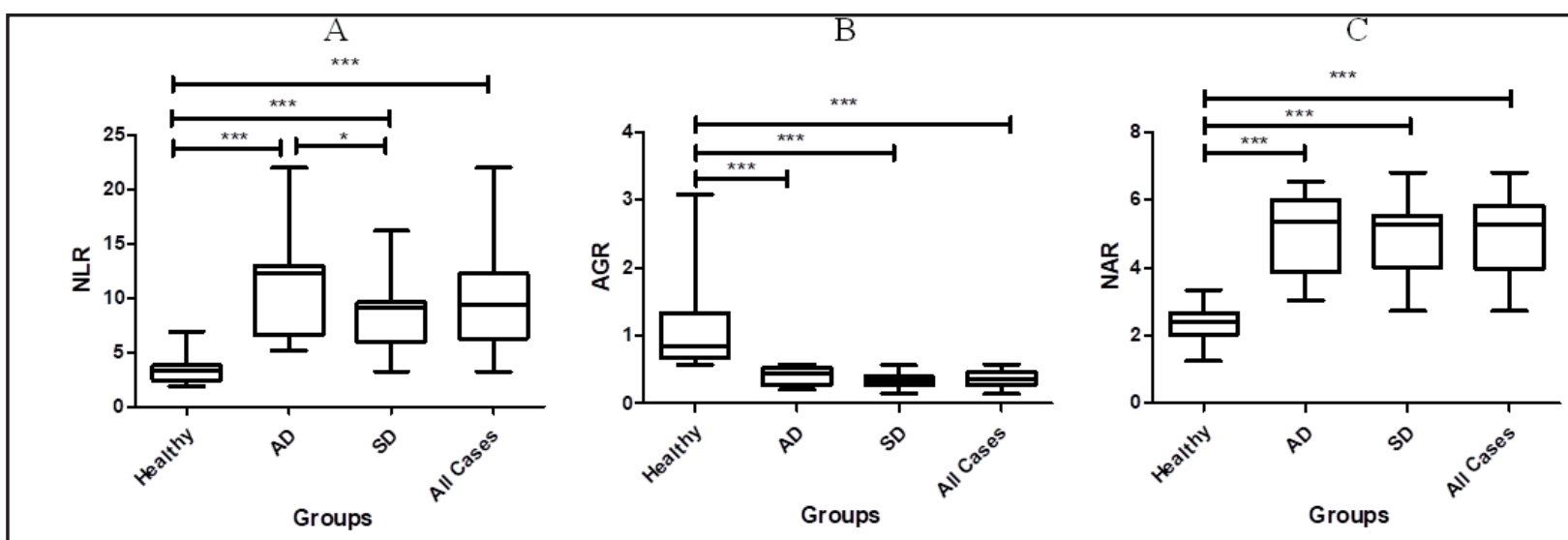

Figure 1. Evaluation of the NLR (A), AGR (B), and NAR (C) parameters in canine leishmaniasis. The lower and upper boundaries of the box represent first and third quartiles of the data, respectively, with the line within the box representing the median. Statistically significant differences are indicated by $*(P<0.05)$ and $* * *(P<0.001)$. NLR, neutrophil-to-lymphocyte ratio; AGR, albumin-to-globulin ratio; NAR: neutrophil-to-albumin ratio; AD: asymptomatic dogs; SD: symptomatic dogs; CD: control dogs; All cases: asymptomatic and symptomatic dogs.

Table 1. Systemic biomarkers in 53 dogs with canine leishmaniasis.

\begin{tabular}{|c|c|c|c|c|c|c|}
\hline \multirow[b]{2}{*}{ Parameter } & \multirow[b]{2}{*}{$\begin{array}{l}\text { Healthy dogs } \\
\qquad(\mathrm{n}=20)\end{array}$} & \multicolumn{3}{|c|}{ CanL } & \multirow[b]{2}{*}{$P$ value } & \multirow[b]{2}{*}{ Reference interval $[10]$} \\
\hline & & $\begin{array}{c}\text { Asymptomatic } \\
(\mathrm{n}=20)\end{array}$ & $\begin{array}{c}\text { Symptomatic } \\
\quad(\mathrm{n}=33)\end{array}$ & $\begin{array}{l}\text { All cases } \\
(\mathrm{n}=53)\end{array}$ & & \\
\hline $\mathrm{WBC}\left(\times 10^{3} / \mathrm{L}\right)$ & $11.49 \pm 2.46$ & $16.48 \pm 4.93^{b}$ & $13.54 \pm 5.13^{\mathrm{c}}$ & $14.65 \pm 5.21^{\mathrm{bc}}$ & 0.0159 & 6 to 17 \\
\hline Absolute neutrophil count $\left(\times 10^{3} / \mathrm{L}\right)$ & $7.35 \pm 1.52$ & $13.41 \pm 3.60$ & $10.42 \pm 3.69^{c}$ & $11.55 \pm 3.91$ & $<0.0001$ & 3 to 11.5 \\
\hline Absolute lymphocyte count $\left(\mathrm{x} 10^{3} / \mathrm{L}\right)$ & $2.36 \pm 0.76$ & $1.31 \pm 0.53$ & $1.30 \pm 0.56$ & $1.30 \pm 0.54$ & $<0.0001$ & 1 to 3.6 \\
\hline NLR & $3.39 \pm 1.19$ & $11.10 \pm 4.36$ & $8.46 \pm 3.00^{c}$ & $9.45 \pm 3.76^{\mathrm{c}}$ & $<0.0001$ & - \\
\hline $\mathrm{STP}(\mathrm{g} / \mathrm{dL})$ & $6.75 \pm 0.54$ & $8.72 \pm 1.21$ & $8.18 \pm 1.04$ & $8.38 \pm 1.12$ & $<0.0001$ & 5.4 to 7.1 \\
\hline Albumin concentration $(\mathrm{g} / \mathrm{dL})$ & $3.20 \pm 0.60$ & $2.48 \pm 0.69$ & $2.10 \pm 0.58^{\mathrm{c}}$ & $2.24 \pm 0.64$ & $<0.0001$ & 2.6 to 3.3 \\
\hline Globulin concentration $(\mathrm{g} / \mathrm{dL})$ & $3.34 \pm 0.76$ & $6.32 \pm 0.87$ & $5.98 \pm 0.87$ & $6.11 \pm 0.88$ & $<0.0001$ & 2.7 to 4.4 \\
\hline AGR & $1.07 \pm 0.58$ & $0.40 \pm 0.13$ & $0.34 \pm 0.10$ & $0.36 \pm 0.11$ & $<0.0001$ & 0.59 to 1.11 \\
\hline NAR & $2.36 \pm 0.55$ & $5.02 \pm 1.14$ & $4.79 \pm 1.07$ & $4.88 \pm 1.09$ & $<0.0001$ & - \\
\hline
\end{tabular}

WBC: White Blood Cells; NLR: Neutrophil to Lymphocyte Ratio; STP: Serum Total Protein; AGR: Albumin to Globulin Ratio; NAR: Neutrophil to Albumin Ratio. Different letters in the same row mean significant difference among treatments $(P<0.05)$. 


\section{DISCUSSION}

A potential link between CanL and inflammation was related on the basis of observations that many animals present inflammatory cells in sites of infection. In human and veterinary medicine, the significance of a variety of systemic inflammation-based prognostic markers has been explored in different cancers $[15,26]$.

In a general view, our results show an increase in circulating leukocytes, neutrophilia, and lymphopenia (Table 1); however, this result is more evident in AD. These data agree with a previous report, whose systemic inflammatory response is associated with leukocyte changes with a reduction in lymphocyte counts in dogs with leishmaniasis $[9,22,36]$. Based on these data, we decided to expand this information using NLR calculation in an attempt to better clarify the changes in these cells in CanL. We observed that NLR was increased on CanL in relation to healthy $\operatorname{dogs}(P<$ $0.0001)$. Here, this result could be as a consequence of relative neutrophilia rather than lymphopenia, demonstrating an important inflammation parameter in CanL. However, on cutaneous leishmaniasis in humans this biomarker is not considered adequate for evaluating systemic inflammation [3].

Neutrophil to Lymphocyte Ratio (NLR) is a biomarker that conveys information about inflammatory conditions. An elevated NLR can reflect an upregulated innate immune response. Neutrophils are effector cells of innate immunity and are involved in several acute and chronic inflammatory processes $[6,41,44]$. Neutrophils migrate from blood vessels to sites invaded by pathogens or affected by other damage, including sterile, under the stimulus of chemoattracting substances and cytokines. These cells are the main phagocytic cells in the immune system and comprise the first line of defense. Recent research places neutrophils as cells that are considered complex due to the diverse functions that they perform besides phagocytosis and for their contribution to the performance of adaptive immunity [18,23,44].

The participation of neutrophils in leishmaniasis disease has been discussed for its active participation in both the capture and the elimination of the protozoan through effective phagocytosis or the release of neutrophil extracellular traps (NETs) [14], oxidative stress [2] or liberation of cytokines, enzymes and others mediators that can contribute to macrophages infection by L. infantum $[19,31,33,39]$. On the other hand, it is known that macrophages are the cells residing in the tissues which are considered the main target cell of L. infantum infection. The participation of neutrophils in the infection of macrophages by L. infantum was observed in an in vitro experiment in which canine neutrophils infected by L. infantum are phagocytosed by macrophages, thus demonstrating the importance of neutrophils in the initial stages of the establishment of CanL [33].

Serum total proteins (STP) and globulins were increased in CanL. In our results it was observed a decrease in serum albumin levels and AGR in CanL, when compared to $\mathrm{CD}$ and references values to canine species. It is already well documented that in CanL there is an increase in the levels of serum globulins mainly associated with the increase of acute phase proteins, cytokines, and increase in the production of specific antibodies in response to this infectious agent $[9,22,37]$.

Reduced plasma albumin levels have been reported in CanL [9]. Albumin is an acute phase protein that is considered an immune-inflammatory biomarker, which can be found reduced systemically in progressive inflammatory response [13,16,20], and has antioxidant properties [39]. This biomarker is used in the medical clinic to assist in the monitoring of inflammatory diseases [4,29]. Our results showed neutrophilia with hypoalbuminemia in CanL. So, in an attempt to assess the relationship between two available markers, we used NAR calculation in order to evaluate the changes in these parameters in CanL. Our data shows that NAR was higher in CanL when compared to control dogs (Table 1). More recently, NAR is used as a predictor of mortality in critical patients with myocardial infarction [8], acute kidney injury [47], severe sepsis or septic shock [12], and bloodstream infection [35]. It is important to report that in CanL the kidneys are targets of damage triggered by the deposit of immune complexes leading to organic failure [43].

In summary, NLR and NAR increase, and AGR decreases in animals with leishmaniasis, demonstrating more explicitly the immune-inflammatory process that occurs in CanL. There is still no data in the recent literature on the use of these parameters in the veterinary medical clinic nor their potential use in the evaluation of inflammatory processes in dogs naturally infected by $L$. infantum. This is the first report that investigated NLR and NAR in CanL. 


\section{CONCLUSION}

Our results indicate that NLR and NAR could be used as biomarkers in the veterinary medical clinic to assess inflammatory profile in CanL, mainly in asymptomatic dogs. Our findings suggest that the NLR, NAR and AGR derived from routine blood tests can be used as clinically meaningful biomarkers to stratify evolution of CanL. Further prospective evaluation of these inflammatory markers is needed through clinical trials in order to validate our findings.

\section{MANUFACTURERS}

'Fundação Oswaldo Cruz - Instituto de Tecnologia em Imunobiológicos (Bio-Manguinhos/Fiocruz). Rio de Janeiro, RJ, Brazil.

${ }^{2}$ Mindray do Brasil - Comércio de Distribuição e Equipamentos Médicos Ltda. São Paulo, SP, Brazil.

${ }^{3}$ Nikon Instruments Inc. Tokyo, Japan.
${ }^{4}$ Labtest Diagnostics S.A. Lagoa Santa, MG, Brazil

${ }^{5}$ GraphPad Software Inc. San Diego, CA, USA.

Funding. This research was supported by National Council for Development and Research (CNPq), Higher Education Personnel Improvement Coordination (CAPES), and Financier of Studies and Projects (FINEP).

Acknowledgements. To the Zoonoses Control Center (ZCC) of Fortaleza/Ceará, especially the Veterinarian Francisco Barroso Pinto for their technical assistance.

Ethical approval. All procedures and animal care were approved by the Ethics Committee for Use of Animals (CEUA) of Universidade Estadual do Ceará (UECE) under protocol number 9166339/2018. All the owners signed an agreement, authorizing the animals' participation in the experiment.

Declaration of interest. The authors declare that they have no conflict of interest. The authors were responsible for the content and writing of the paper.

\section{REFERENCES}

1 Abbehusen M.M.C., Almeida V.D.A., Solcà M.D.S., Pereira L.S., Costa D.J., Gil-Santana L., Bozza P.T., Fraga D.B.M., Veras P.S.T., Santos W.L.C., Andrade B.B. \& Brondskyn C.I. 2017. Clinical and immunopathological findings during long term follow-up in Leishmania infantum experimentally infected dogs. Scientific Reports. 7(1): 15914.

2 Almeida B.F.M., Narciso L.G., Melo L.M., Preve P.P., Bosco A.M., Lima V.M.F. \& Ciarlini P.C. 2013. Leishmaniasis causes oxidative stress and alteration of oxidative metabolism and viability of neutrophils in dogs. The Veterinary Journal. 198(3): 599-605.

3 An I., Ayhan E., Aksoy M., Erat T. \& Doni N.Y. 2021. Evaluation of inflammatory parameters in patients with cutaneous leishmaniasis. Dermatologic Therapy. 34(1): e14603.

4 Artigas A., Wernerman J., Arroyo V., Vincent J.L. \& Levy M. 2016. Role of albumin in diseases associated with severe systemic inflammation: pathophysiologic and clinical evidence in sepsis and in decompensated cirrhosis. Journal of Critical Care. 33: 62-70.

5 Cantos-Barreda A., Escribano D., Martínez-Subiela S., Pardo-Marin L., Segarra S. \& Céron J.J. 2018. Changes in serum anti-Leishmania antibody concentrations measured by time-resolved immunofluorometric assays in dogs with leishmaniosis after treatment. Veterinary Immunology and Immunopathology. 198: 65-69.

6 Cascao R., Rosario H.S. \& Fonseca J.E. 2009. Neutrophils: warriors and commanders in immune mediated inflammatory diseases. Acta Reumatológica Portuguesa. 34(2B): 313-326.

7 Chagas A.C., Oliveira F., Debrabant A., Valenzuela J.G., Ribeiro J.M.C. \& Calvo E. 2014. Lundep, a sand fly salivary endonuclease increases Leishmania parasite survival in neutrophils and inhibits xiia contact activation in human plasma. PLoS Pathogens. 10(2): e1003923.

8 Cui H., Ding X., Li W., Chen H. \& Li H. 2019. The neutrophil percentage to albumin ratio as a new predictor of inhospital mortality in patients with st-segment elevation myocardial infarction. Medical Science Monitor. 25: 7845-7852.

9 Freitas J.C.C., Nunes-Pinheiro D.C.S., Lopes Neto B.E., Santos G.J.L., Abreu C.R.A., Braga R.R., Campos R.M. \& de Oliveira L.F. 2012. Clinical and laboratory alterations in dogs naturally infected by Leishmania chagasi. Revista da Sociedade Brasileira de Medicina Tropical. 45(1): 24:29.

10 Freeman P.K. \& Klenner S. 2015. Veterinary clinical pathology. A case-based approach. Boca Raton: CRS Press, 288p.

11 Gabriel C., McMaster W.R., Girard D. \& Descoteaux A. 2014. Leishmania donovani promastigotes evade the antimicrobial activity of neutrophil extracellular traps. Journal of Immunology. 185(7): 4319-4327.

12 Gong Y., Li D., Cheng B., Ying B. \& Wang B. 2020. Increased neutrophil percentage-to-albumin ratio is associated with all-cause mortality in patients with severe sepsis or septic shock. Epidemiology and Infection. 148: e8. 
13 Gradel K.O., Vinholt P.J., Magnussen B., Pedersen C., Jensen T.G., Kolmos H.J. \& Lassen A.T. 2018. Hypoalbuminaemia as a marker of trans-capillary leakage in community-acquired bacteraemia patients. Epidemiology and Infection. 146(5): 648-655.

14 Guimarães-Costa A.B., Nascimento M.T.C., Froment G.S., Soares R.P.P., Morgado F.N., Conceição-Silva F. \& Saraiva E.M. 2009. Leishmania amazonensis promastigotes induce and are killed by neutrophil extracellular traps. Proceedings National Academy of Sciences. 106(16): 6748-6753.

15 Guthrie G.J., Charles K.A., Roxburgh C.S.D., Horgan P.G., McMillan D.C. \& Clarke S.J. 2013. The systemic inflammation-based neutrophil-lymphocyte ratio: experience in patients with cancer. Critical Review in Oncology/ Hematology. 88(1): 218-30.

16 Ha C.E. \& Bhagavan N.V. 2013. Novel insights into the pleiotropic effects of human serum albumin in health and disease. Biochimica et Biophysica Acta. 1830(12): 5486-5493.

17 Hodgson N., Llewellyn E.A. \& Schaeffer D.J. 2018. Utility and prognostic significance of neutrophil-to-lymphocyte ratio in dogs with septic peritonitis. Journal of the American Animal Hospital Association. 54(6): 351-359.

18 Hsu B.E., Shen Y. \& Siegel P.M. 2020. Neutrophils: orchestrators of the malignant phenotype. Frontiers in Immunology. 11: 1778.

19 Hurrell B.P., Regli I.B. \& Tacchini-Cottier F. 2016. Different Leishmania species drive distinct neutrophil functions. Trends in Parasitology. 32(5): 392-401.

20 Kinoshita H., Watanabe K., Azma T., Feng G.G., Akahori T., Hayashi H., Sato M., Fujiwara Y. \& Wakatsuki A. 2017. Human serum albumin and oxidative stress in preeclamptic women and the mechanism of albumin for stress reduction. Heliyon. 3(8): e00369.

21 Laurenti M.D., Rossi C.N., da Matta V.L.R., Tomokane T.Y., Corbett C.E.P., Secundino N.F.C., Pimenta P.F.P. \& Marcondes M. 2013. Asymptomatic dogs are highly competent to transmit Leishmania (Leishmania) infantum chagasi to the natural vector. Veterinary Parasitology. 196(3-4): 296-300.

22 Leal G.G.A., Roatt B.M., Aguiar-Soares R.D.O., Carneiro C.M., Giunchetti R.C., Teixeira-Carvalho A., Martins Filho O.A., Francisco A.F., Cardoso J.M., Mathias F.A.S., Correa-Oliveira R., Carneiro M., Coura-Vital W. \& Reis A.B. 2014. Immunological profile of resistance and susceptibility in naturally infected dogs by Leishmania infantum. Veterinary Parasitology. 205(3-4): 472-482.

23 Liew P.X. \& Kubes P. 2019. The neutrophil's role during health and disease. Physiological Reviews. 99(2): 1223-1248.

24 Macfarlane L., Morris J., Pratschke K., Mellor D., Scase T., Macfarlane M. \& Mclauchlan G. 2015. Diagnostic value of neutrophil-lymphocyte and albumin-globulin ratios in canine soft tissue sarcoma. Journal of Small Animal Practice. 57(3): 135-141.

25 Macfarlane M.J., Macfarlane L.L., Scase T., Parkin T. \& Morris J.S. 2016. Use of neutrophil to lymphocyte ratio for predicting histopathological grade of canine mast cell tumours. Veterinary Records. 179(19): 1-6.

26 Martin H.L., Ohara K., Kiberu A., Hagen T.V., Davidson A. \& Khattak M.A. 2014. Prognostic value of systemic inflammation-based markers in advanced pancreatic cancer. Internal Medicine Journal. 44(7): 676682.

27 Martínez-Orellana P., Marí-Martorell D., Montserrat-Sangrà S., Ordeix L., Baneth G. \& Solano-Gallego L. 2017. Leishmania infantum-specific IFN- $\gamma$ production in stimulated blood from dogs with clinical leishmaniosis at diagnosis and during treatment. Veterinary Parasitology. 248: 39-47.

28 Mutz M., Boudreaux B., Kearney M., Stroda K., Gaunt S. \& Shiomitsu K. 2013. Prognostic value of baseline absolute lymphocyte concentration and neutrophil/lymphocyte ratio in dogs with newly diagnosed multi-centric lymphoma. Veterinary and Comparative Oncology. 13(4): 337-347.

29 Norberg Å., Rooyackers O., Segersvärd R. \& Wernerman J. 2015. Albumin kinetics in patients undergoing major abdominal surgery. PLoS One. 10(8): e0136371.

30 Oikonomidis I.L., Tsouloufi T.K., Mylonakis M.E., Psalla D., Soubasis N., Rallis T. \& Kritsepi-Konstantinou M. 2019. Circulating and bone marrow myeloid cells containing Leishmania amastigotes in a case of advanced canine leishmaniosis. Journal of Veterinary of Diagnostic Investigation. 31(5): 726-731.

31 Oualha R., Barhoumi M., Marzouki S., Harigua-Souiai E., Ahmed M.B. \& Guizani I. 2019. Infection of human neutrophils with Leishmania infantum or Leishmania major strains triggers activation and differential cytokines release frontier in cellular and infection. Microbiology. 9: 153. 
32 Peters N.C., Egen J.G., Secundino N., Debrabant A., Kimblin N., Kamhawi S., Lawyer P., Fay M.P., Germain R.N. \& Sacks D. 2008. In vivo imaging reveals an essential role for neutrophils in leishmaniasis transmitted by sand flies. Science. 321(5891): 970-974.

33 Pereira M.A., Alexandre-Pires G., Câmara M., Santos M., Martins C., Rodrigues A., Adriana J., Passero L.F.D., Da Fonseca I.P. \& Santos-Gomes G. 2019. Canine neutrophils cooperate with macrophages in the early stages of Leishmania infantum in vitro infection. Parasite Immunology. 41(4): e12617.

34 Pierini A., Gori E., Lippi I., Ceccherini G., Lubas G. \& Marchetti V. 2019. Neutrophil-to-lymphocyte ratio, nucleated red blood cells and erythrocyte abnormalities in canine systemic inflammatory response syndrome. Research in Veterinary Science. 126: 150-154.

35 Qu J., Yuan H.-Y., Huang Y., Qu Q., Ou-Yang Z.-B., Li G.-H., Zhu H.-H. \& Lu Q. 2019. Evaluation of neutrophillymphocyte ratio in predicting bloodstream infection. Biomarkers in Medicine. 13(15): 1255-1261.

36 Reis A.B., Martins Filho O.A., Teixeira-Carvalho A., Carvalho M.G., Mayrink W., França-Silva J.C., Giunchetti R.C., Genaro O. \& Corrêa-Oliveira R. 2006. Parasite density and impaired biochemical/hematological status are associated with severe clinical aspects of canine visceral leishmaniasis. Research in Veterinary Science. 81(1): 68-75.

37 Reis A.B., Martins Filho O.A., Teixeira-Carvalho A., Giunchetti R.C., Carneiro C.M., Mayrink W., Tafuri W.L. \& Corrêa-Oliveira R. 2009. Systemic and compartmentalized immune response in canine visceral leishmaniasis. Veterinary Immunology and Immunopathology. 128(1-3): 87-95.

38 Ribeiro R.R., Silva S.M., Fulgêncio G.O., Michalick M.S.M. \& Frézard F.J.G. 2013. Relationship between clinical and pathological signs and severity of canine leishmaniasis. Revista Brasileira de Parasitologia Veterinária. 22(3): 373-378.

39 Ribeiro-Gomes F.L. \& Sacks D. 2012. The influence of early neutrophil-Leishmania interactions on the host immune response to infection. Frontiers in Cellular and Infection Microbiology. 2: 59.

40 Roche M., Rondeau P., Singh N.R., Tarnus E. \& Bourdon E. 2008. The antioxidant properties of serum albumin. Febs Letters. 582(13): 1783-1787.

41 Rosales C. 2020. Neutrophils at the crossroads of innate and adaptive immunity. Journal of Leukcyte Biology. 108(1): 377-396.

42 Rossaint J., Margraf A. \& Zarbock A. 2018. Role of platelets in leukocyte recruitment and resolution of inflammation. Frontiers in Immunology. 9: 1-13.

43 Roura X., Cortadellas O., Day M.J., Benali S.L., Group C.L.W. \& Zatelli A. 2020. Canine leishmaniosis and kidney disease: Q\&A for an overall management in clinical practice. Journal of Small and Animal Practice. 62(1): E1-E19.

44 Sadik C.D., Kim N.D. \& Luster A.D. 2011. Neutrophils cascading their way to inflammation. Trends in Immunology. 32(10): 452-460.

45 Solano-Gallego L., Koutinas A., Miró G., Cardoso L. \& Pennisi M.G. 2009. Directions for the diagnosis, clinical staging, treatment and prevention of canine leishmaniasis. Veterinary Parasitology. 165(1-2): 1-18.

46 Verçosa B.L.A., Muniz-Junqueira M.I., Menezes-Souza D., Fujiwara R.T., Melo M.N. \& Vasconcelos A.C. 2021. The balance between IL-12/IL4 in renal tissue switches the inflammatory response arm and shows relationship with the clinical signs in Leishmania-infected dogs. Veterinary Immunology and Immunopathology. 234: 110196.

47 Wang B., Li D., Cheng B., Ying B. \& Gong Y. 2020. The neutrophil percentage-to-albumin ratio is associated with all-cause mortality in critically ill patients with acute kidney injury. BioMed Research International. 18: 5687672. 